\title{
Análisis espacial del riesgo de establecimiento de Lymantria dispar en bosques nativos de Sudamérica
}

\author{
Spatial analyses of risk establishment for Lymantria dispar in South American native forests \\ Guillermo Heit $^{\mathrm{a}, \mathrm{b} *}$, Ana Iribarne ${ }^{\mathrm{a}}$, Walter Sione ${ }^{\mathrm{c}, \mathrm{d}}$, Pablo Aceñolaza ${ }^{\mathrm{c}}$, Pablo Cortese ${ }^{\mathrm{a}}$ \\ a Senasa, Dirección de Vigilancia y Monitoreo, Ciudad Autónoma de Buenos Aires, Argentina.

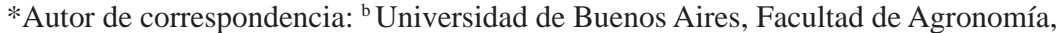 \\ Ciudad Autónoma de Buenos Aires, tel.:5445248046, gheit@agro.uba.ar \\ 'Universidad Autónoma de Entre Ríos-CONICET, CEREGEO, Entre Ríos, Argentina. \\ ${ }^{d}$ Universidad Nacional de Luján, PRODITEL, Buenos Aires, Argentina.
}

\begin{abstract}
SUMMARY
Lymantria dispar is a quarantine pest absent in South America. A regional phytosanitary surveillance plan was developed to control this pest due to its high risk of introduction through global commerce. The objective of this study was to estimate the potential distribution ranges of $L$. dispar in a new geographical area, using a deductive approach to prioritize areas for preventive monitoring. In South America, forest susceptibility was evaluated under different climate scenarios. In Argentina a phenology model was simulated. According to historical climatic conditions in South America, the areas with higher risk of establishment are the Andean forests from northern Argentina to Venezuela, the Brazilian Atlantic forest, littoral coastal areas of Uruguay and Argentina and the Mediterranean area of central and southern Chile. Based on estimations of IPCC, the effects of climate change would cause a reduction of the susceptible area to L. dispar invasion in future decades. Although there were statistically significant differences among the areas with high risk of invasion, estimated by the two methodological approaches, susceptible areas for preventive monitoring were identified.
\end{abstract}

Key words: invasive species, climate change, phytosanitary risk.

\section{RESUMEN}

Lymantria dispar es una plaga cuarentenaria ausente de Sudamérica, para la que se ha elaborado un plan de vigilancia fitosanitaria regional, debido su elevado riesgo de introducción involuntaria a través del comercio. El objetivo de este trabajo fue estimar la distribución geográfica potencial de L. dispar en una nueva área utilizando un enfoque deductivo, a fin de priorizar ambientes en riesgo para su monitoreo preventivo. En Sudamérica se evaluó la susceptibilidad de la cobertura boscosa ante diferentes escenarios climáticos. A escala local, en Argentina, se simuló la fenología potencial de la especie. A base de las condiciones climáticas históricas de Sudamérica, L. dispar podría establecerse en los bosques andinos desde el norte de Argentina, hasta Venezuela, la mata atlántica brasileña, áreas costeras litorales de Uruguay y Argentina y el área mediterránea del centro y sur de Chile. Los efectos del cambio climático estimados por el IPCC, provocarían una disminución de este área susceptible en las próximas décadas. Aunque existieron diferencias significativas en la superficie clasificada como de mayor riesgo fitosanitario en las dos escalas de estudio (subcontinental vs. regional), se lograron identificar las principales áreas susceptibles para el monitoreo preventivo de L. dispar.

Palabras clave: especie invasora, cambio climático, riesgo fitosanitario.

\section{INTRODUCCIÓN}

El fuerte aumento del comercio internacional observado en las últimas décadas ha incrementado la probabilidad de que muchas especies se establezcan fuera de su área de distribución natural (Levine y D’Antonio 2003). Lymantria dispar L. (Lepidoptera: Lymantriidae), es una plaga cuarentenaria que, hasta el presente, se encuentra ausente de Sudamérica. La misma es considerada una amenaza fitosanitaria para los bosques nativos y plantaciones forestales de latifoliadas, debido al riesgo potencial de introducción involuntaria de la misma a través del comercio global (Tobin et al. 2012).
Este lepidóptero univoltino, defoliador, se asocia a unas 250 especies de hospederos vegetales, fundamentalmente árboles latifoliados, en su actual rango de distribución (Liebhold et al. 2000).

El Comité de Sanidad Vegetal del Conosur (COSAVE), ha elaborado un plan de vigilancia fitosanitaria regional, por el que cada uno de los países integrantes, se compromete a establecer un sistema de detección precoz de la especie (COSAVE 2000). En tal sentido, la construcción de un modelo de distribución potencial de la especie, podría auxiliar las tareas de planificación estratégica de los sistemas de vigilancia en cada uno de los países miembros. El objetivo del presente trabajo fue simular la distribución geográfica potencial de 
L. dispar, utilizando un enfoque deductivo, a fin de priorizar ambientes en riesgo para el monitoreo preventivo.

\section{MÉTODOS}

Se estimó la distribución potencial de L. dispar a dos escalas espaciales de estudio. A escala subcontinental se tomó como área de estudio a Sudamérica y a escala regional, se tomó como caso la República Argentina. En ambas escalas de estudio se utilizó un enfoque metodológico deductivo para clasificar los ambientes con riesgo de establecimiento (R), en función de las condiciones climáticas predisponentes (C), la distribución de hospederos vegetales (H) y la accesibilidad para la especie (A). Siendo entonces $\mathrm{R}=\mathrm{C} \cap \mathrm{H} \cap \mathrm{A}$ (Soberón y Peterson 2005). El detalle de las variables utilizadas en el análisis se presenta en el cuadro 1.

Estimación de ambientes climáticamente favorables (C). La estimación de los ambientes favorables para el establecimiento de la especie, difirió metodológicamente entre las diferentes escalas de estudio.

A escala subcontinental, se compararon diferentes escenarios climáticos, mediante la utilización de estadísticas meteorológicas mensuales. Se consideró un escenario climático actual, basado las estadísticas meteorológicas históricas 1950-2000. Tres escenarios futuros (décadas de 2040, 2060 y 2080), estimados por The Intergovernmental Panel on Climate Change (IPCC), en función de requerimientos energéticos globales moderados, SERES A1B (Ramirez y Jarvis 2008). Por último, con finalidad metodológica, se construyó un hipotético escenario de disminución de $1^{\circ} \mathrm{C}$ en la temperatura media de Sudamérica.
Los parámetros bioecológicos de la especie se obtuvieron del plan de vigilancia de COSAVE (2000), corregidos por Vanhanen et al. (2007) (figura 1).

A escala regional, se utilizó una base de datos climáticos con una mayor resolución espacial y menor escala temporal que la utilizada a escala subcontinental (cuadro 1). Esto permitió simular la fenología potencial de la especie, en base a los parámetros del Gypsy Moth Phenology Model (GMPHEM, Sheehan 1992), por medio de estadísticas meteorológica de paso diario del Servicio Meteorológico Nacional de Argentina (Blanco et al. 2012).

El modelo GMPHEM utiliza las temperaturas máximas y mínimas diarias para calcular el tiempo térmico requerido en cada estado de desarrollo de la especie. Aunque el modelo ha sido ampliamente utilizado, debemos remarcar que el mismo no incorpora la variabilidad climática de microescala, como así también la variabilidad fenológica (Andersen et al. 2001).

En primer lugar, se asumió que los días de frio acumulados en un ambiente dado determinan, la cantidad de cohortes potenciales en que puede dividirse el periodo de eclosión de huevos y se realizó una primera clasificación del ambiente, en función de tal variabilidad. Posteriormente, se realizaron 28 iteraciones del modelo (denominadas cohortes), entre el 1 de julio y el 31 de marzo. Se supuso que cada cohorte de larvas eclosionó 23 días después que la precedente (figura 2). Posteriormente, en función de un modelo aditivo, se clasificaron los ambientes en función de los requerimientos de frío de la especie y la estacionalidad del vuelo de adultos, a menor variabilidad de eclosión de huevos y adultos, mayor riesgo de establecimiento. La fecha estimada de vuelo del 50 \% de la población de adultos

Cuadro 1. Base de datos incluida en el análisis. Datasets included in analyses.

\begin{tabular}{|c|c|c|c|c|c|}
\hline Variable & Tipo & Fuente & Resolución & Referencia & Área de análisis \\
\hline $\begin{array}{l}\text { Índice de vegetación } \\
\text { normalizado máximo } \\
\text { mensual }\end{array}$ & Vegetación & $\begin{array}{l}\text { NDVI, MOD13Q1 } \\
(2000-2009)\end{array}$ & $250 \mathrm{~m}$ & $\begin{array}{c}\text { NASA Land Processes } \\
\text { Distributed Active Center }\end{array}$ & $\begin{array}{l}\text { Sudamérica/ } \\
\text { Argentina }\end{array}$ \\
\hline $\begin{array}{l}\text { Tipo de cobertura } \\
\text { vegetal }\end{array}$ & Vegetación & $\begin{array}{l}\text { Globcover/ } \\
\text { ESA }\end{array}$ & $300 \mathrm{~m}$ & Bicheron et al. 2006 & $\begin{array}{l}\text { Sudamérica/ } \\
\text { Argentina }\end{array}$ \\
\hline $\begin{array}{l}\text { Temperatura media } \\
\text { mensual }\end{array}$ & Climática & Worldclim (1950-2000) & $10 \mathrm{~km}$ & Hijmans et al. 2005 & Sudamérica \\
\hline $\begin{array}{l}\text { Temperatura media } \\
\text { mensual (2040, } \\
\text { 2060, 2080) }\end{array}$ & Climática & IPCC, SERES A1B & $10 \mathrm{~km}$ & Ramírez y Jarvis 2008 & Sudamérica \\
\hline $\begin{array}{l}\text { Temperatura media } \\
\text { diaria }\end{array}$ & Climática & $\begin{array}{l}\text { Interpolación espacial } \\
\text { datos SMN (1990-2009) }\end{array}$ & $4 \mathrm{~km}$ & Blanco et al. 2010 & Argentina \\
\hline $\begin{array}{l}\text { Distancia al puerto } \\
\text { más cercano }\end{array}$ & Antropogénica & Elaboración propia & $4 \mathrm{~km}$ & $* * *$ & $\begin{array}{l}\text { Sudmérica/ } \\
\text { Argentina }\end{array}$ \\
\hline
\end{tabular}

IPCC: The Intergovernmental Panel on Climate Change.

SMN: Servicio Meteorológico Nacional (Argentina).

MOD13Q1: Índice de vegetación MODIS (16 días). ASTER L1B. USGS/Earth Resources Observation and Science

(EROS) Center, Sioux Falls, South Dakota. 2001 (https://lpdaac.usgs.gov/data_access). 


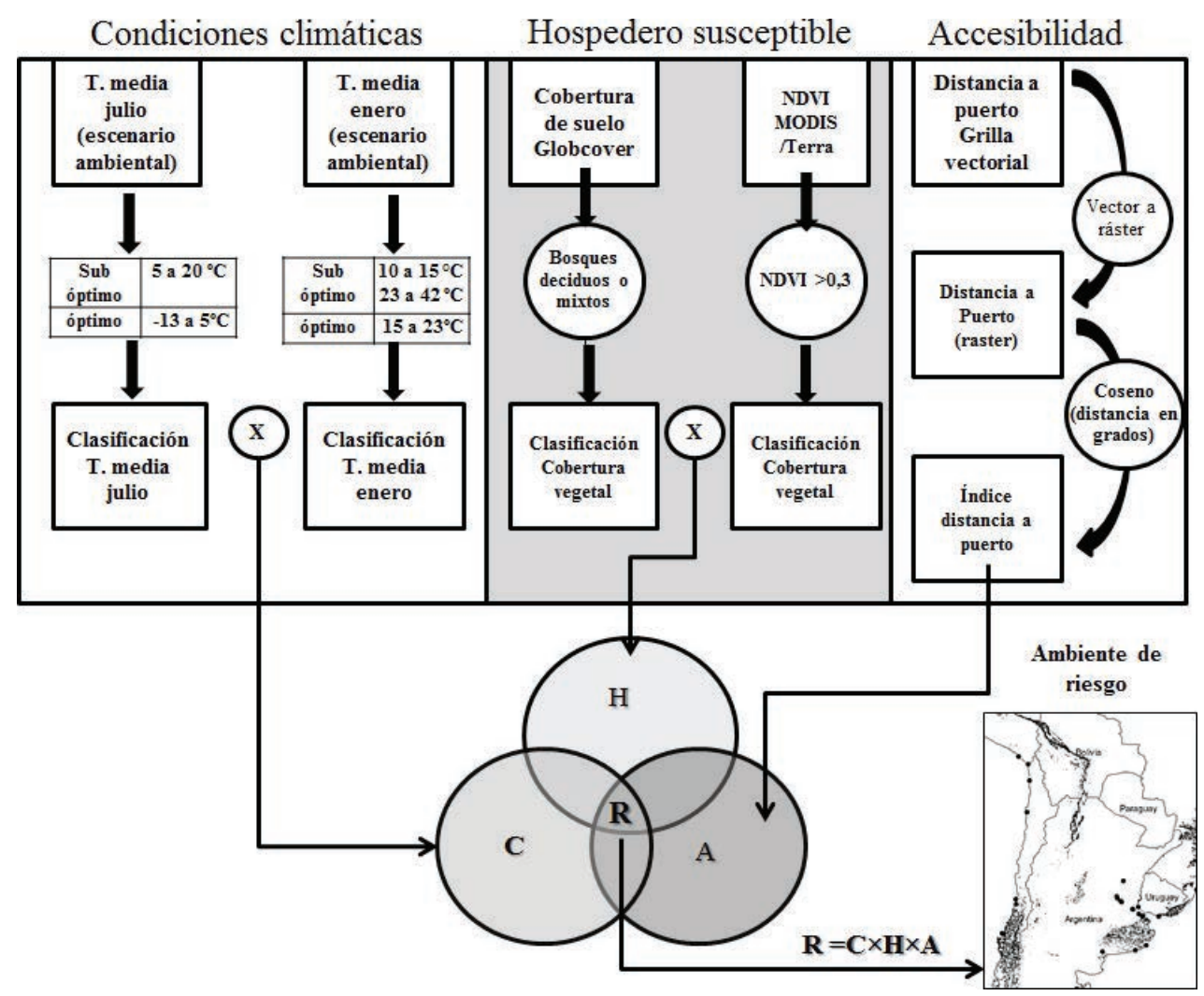

Figura 1. Diagrama general de asociación de variables para el análisis espacial del riesgo de plagas.

General diagram of spatial association of variables for pest risk analyses.

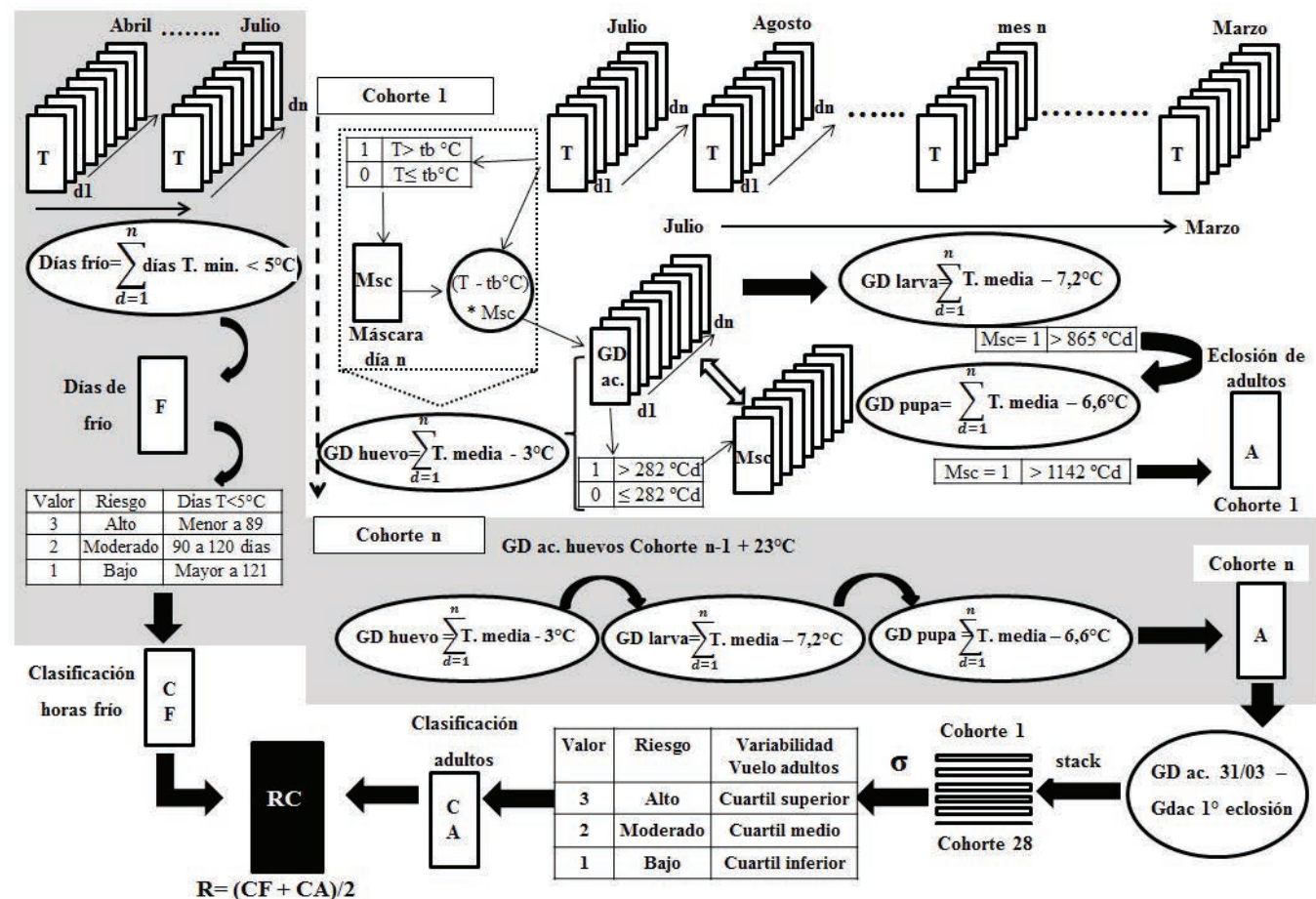

Figura 2. Diagrama general de asociación de variables climáticas diarias para simular la fenología de L. dispar en Argentina. Abreviaturas incluidas en el diagrama: $\mathrm{T}$ = temperatura media diaria, GD = grados día, Msc.= máscara.

General diagram of daily climatic variables association to simulate the phenology of $L$. dispar at regional scale. Abbreviations included in the diagram: $\mathrm{T}=$ mean daily temperature, $\mathrm{GD}=$ degree day, Msc. = mask. 
se calculó mediante la consulta espacial de una muestra aleatoria de 20 sitios, por ecorregión, con cobertura vegetal susceptible.

Distribución de hospederos (C). La distribución geográfica de hospederos susceptibles en Sudamérica y Argentina, se discriminó a partir de la capa de cobertura vegetal GLOBCOVER-ESA, al seleccionar las clases que contienen bosques deciduos o mixtos (Sharov et al. 1999). Dentro de estas clases, se seleccionaron áreas con NDVI mensual mayor a 0,3 , para los meses de octubre a marzo. A fin de descartar celdas con un elevado porcentaje de suelo descubierto en la época de activo crecimiento de especies latifoliadas.

Accesibilidad de L. dispar (A). Zlotina et al. (1999) hallaron que las larvas que eclosionan a partir de las oviposiciones de $L$. dispar, pueden ser transportadas por el viento a distancias mayores a $2 \mathrm{~km}$, favorecidas por la formación de hilos de seda. Este mecanismo de dispersión posee impacto en la dispersión a cortas distancias, pero a largas distancias, el mecanismo más frecuente de dispersión, es el transporte antrópico. Entre estos, la vía de introducción más probable de la especie a Sudamérica, la constituye el transporte de masas de huevos viables adheridos a las cubiertas de los barcos o contenedores (Gadgil et al. 2000). En este sentido, se consideró como áreas con mayor riesgo de ser inicialmente invadidas, aquellas ubicadas dentro de un radio de $2 \mathrm{~km}$ de las instalaciones portuarias. Para el resto de la cobertura boscosa/forestal del subcontinente, se ponderó el riesgo de accesibilidad de la especie, a base del cálculo de una variable de mínima distancia a los potenciales sitios de ingreso (puertos).

Análisis de sensibilidad. Se realizó un análisis de sensibilidad a fin de estimar la estabilidad del modelo, evaluando la variación provocada en los resultados del mismo en función de variaciones sistemáticas sobre los parámetros de entrada. Para ello, se calculó un índice (S) de diferencia media en la superficie de riesgo entre el modelo modificado y el de referencia (modificado de Orán Cáseres et al. 2011). Este índice se calculó como:

$$
\begin{gathered}
\mathrm{S}=\left(\sum_{\mathrm{i}} \mid \mathrm{P}_{\mathrm{ci}} \text { capa original }-\mathrm{P}_{\mathrm{ci}} \text { capa modificada } \mid /\right. \\
\left.\mathrm{P}_{\mathrm{ci}} \text { capa original }\right) / \mathrm{N}^{\circ} \text { iteraciones. }
\end{gathered}
$$

Dónde:

$\mathrm{S}=$ diferencia media en la superficie de riesgo que existe entre el mapa original y el mapa modificado; $\mathrm{P}=$ número de pixeles de la categoría ci.

Se realizaron 20 iteraciones del modelo, contemplando en cada una de ellas, modificaciones de un $1 \%$ en los valores de entrada en los parámetros originales de temperatura y NDVI, hasta lograr una modificación global de $\pm 10 \%$ en los mismos.
La variabilidad intrínseca de las variables de entrada al modelo fenológico se evaluó mediante la estimación de la raíz del error cuadrático medio (RMSE), de cada pixel, entre el 1 de abril y el 31 de marzo, a base de la fórmula (Liu et al. 2009):

RMSE $=\sqrt{\frac{\sum_{i=1}^{n}\left(X_{\text {obs,i }}-X_{\text {model }, i}\right)^{2}}{n}}$

Dónde:

$\mathrm{X}_{\mathrm{obs}, \mathrm{i}}=$ valor observado en el pixel/momento i

$\mathrm{X}_{\text {model }, \mathrm{i}}=$ valor modelado en el pixel $/$ momento i

$\mathrm{n}$ =día calendario entre el 1 abril y el 31 de marzo.

Para el tratamiento de la información se utilizó el software GVSIG ${ }^{\circledR} 1.11$ y Qgis 1.8 .

\section{RESULTADOS}

Como puede observarse en la figura 3 , con base en las condiciones climáticas históricas de Sudamérica, L. dispar podría potencialmente establecerse en los bosques andinos desde el norte de Argentina, hasta Venezuela, la mata atlántica brasileña, áreas costeras litorales de Uruguay y Argentina y área mediterránea húmeda y prehúmeda del centro y sur de Chile.

Según las estimaciones del IPCC, los efectos del cambio climático provocarían una disminución del área susceptible a ser invadida por $L$. dispar en Sudamérica $(P=0,01)$ (figura 3). Se estimó un aumento de la cobertura boscosa con condiciones climáticamente desfavorables para el desarrollo de esta especie de hasta $67.000 \mathrm{~km}^{2}$, por cada grado centígrado de incremento de temperatura hacia el 2080. Asimismo es de esperar una disminución del área de moderado riesgo de establecimiento, de $50.000 \mathrm{~km}^{2} /{ }^{\circ} \mathrm{C}$ $(P=0,01)$. Sin embargo, se prevé un leve aumento de la superficie considerada de riesgo elevado $\left(4.000 \mathrm{~km}^{2} /{ }^{\circ} \mathrm{C}\right.$, $P=0,04$ ) (cuadro 2).

La diferencia media entre las superficies de riesgo estimadas por el modelo de referencia y las variaciones sistemáticas de los parámetros de entrada al mismo, índice $\mathrm{S}$, se muestran en el cuadro 3. En el mismo puede observarse que la clasificación inicial del factor temperatura media mensual y la clasificación de ambientes en función de áreas de aptitud climática (fase 2), son muy sensibles a la modificación planteada en su modelación. El modelo se mostró más estable en las dos primeras fases del análisis utilizando las estadísticas climáticas históricas, que utilizando los escenarios climáticos futuros calculados por el IPCC. La clasificación de coberturas vegetales con aptitud climática para el establecimiento de $L$. dispar, se mostró muy estable a los cambios sistemáticos realizados con cada iteración de análisis de sensibilidad.

Al simular la fenología a escala regional, se encontró que las áreas con mayor susceptibilidad de colonización se hallan distribuidas en las ecorregiones de los Bosques 


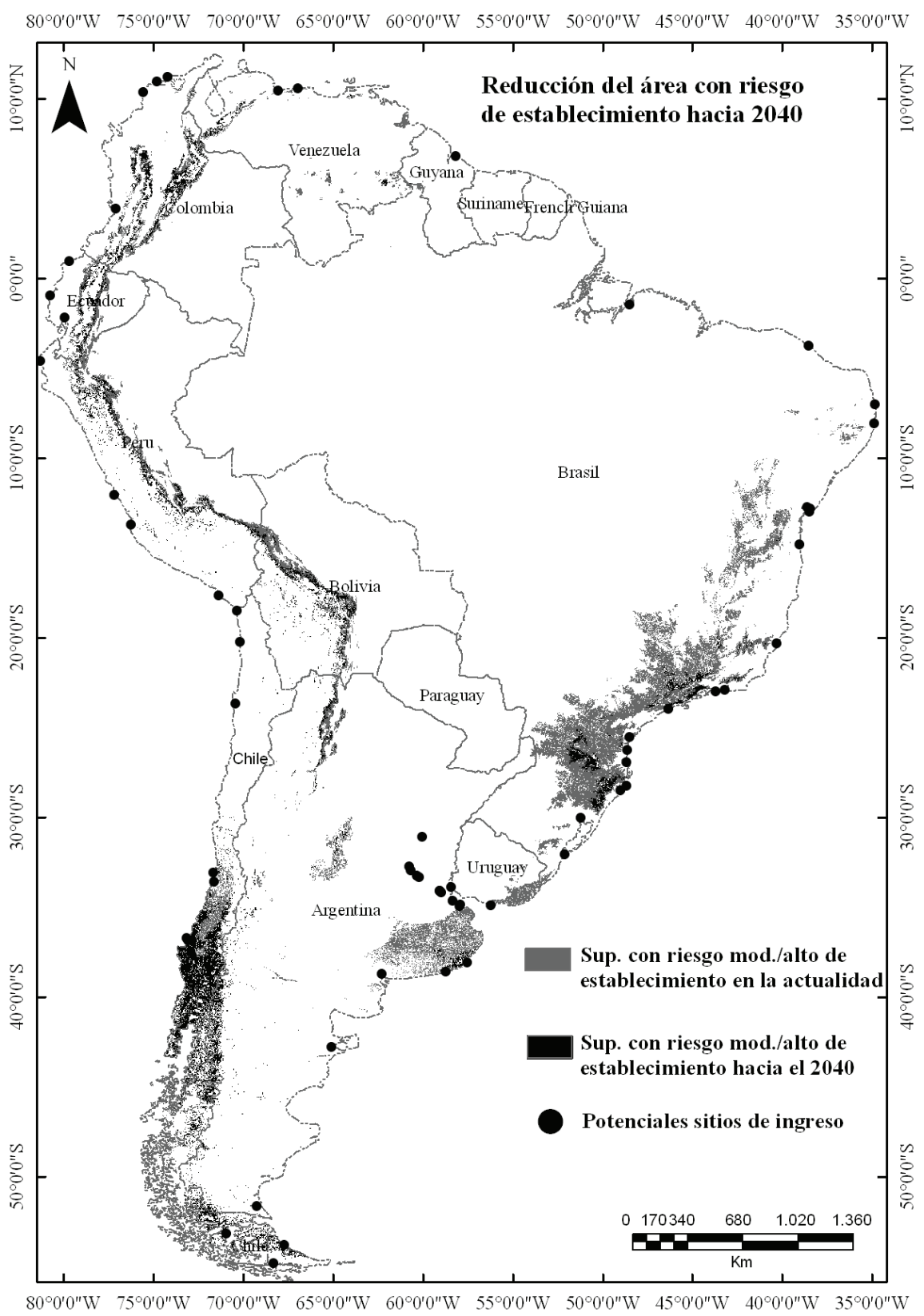

Figura 3. Comparación de las áreas con moderado a elevado riesgo de establecimiento de $L$. dispar en Sudamérica, con base en las estadísticas climáticas históricas y las estimaciones climáticas del IPCC para la década de 2040.

Comparison of areas with moderate to high risk of establishment for L. dispar in South America based on historical climatic statistics and IPCC climate projections for the 2040s.

Patagónicos, la Pampa, el Delta del Paraná y la Selva de Yungas (figura 4).

Esta zonificación general del riesgo es similar a la encontrada mediante el análisis espacial a escala subcontinental, pero mostró diferencias estadísticamente significativas en las superficies estimadas para cada una las categorías de riesgo $\left(G_{(3,0,05)}^{2}=18,03 ; P=0,0004\right)$. Mediante la simulación fenológica de la especie en Argentina se estimó que la superficie de cobertura boscosa/forestal con moderado a elevado riesgo de establecimiento de $L$. dispar es un $4 \%$ mayor que la estimada por el modelo deductivo de escala subcontinental y paso mensual, mientras que el área sin riesgo es un $11 \%$ mayor.

Según el modelo fenológico, las ecorregiones que presentarían una mayor superficie categorizada como de elevado riesgo de establecimiento serían los Bosques Patagónicos y la Pampa, con un 44 y 39 \% de participación relativa, respectivamente (cuadro 4). Sin embargo, los datos climáticos utilizados para la simulación fenológica presentan un mayor error de estimación intrínseco para la 
Cuadro 2. Variación de la superficie potencial con riesgo de establecimiento de $L$. dispar, en función de diferentes escenarios climáticos. Variation of the area with potential risk of establishment for $L$. dispar, according to different climate scenarios.

\begin{tabular}{|c|c|c|c|c|c|c|c|c|c|c|}
\hline \multirow{2}{*}{$\begin{array}{c}\text { Caracterización de } \\
\text { ambientes }\end{array}$} & \multirow{2}{*}{ Actual } & \multicolumn{3}{|c|}{ Escenarios futuros } & \multirow{2}{*}{$\begin{array}{c}\text { Hipótesis } \\
\text { enfriamiento }\end{array}$} & \multirow{2}{*}{$m$} & \multirow{2}{*}{$F$} & \multirow{2}{*}{$P$} & & \multirow{2}{*}{$R^{2}$} \\
\hline & & 2040 & 2060 & 2080 & & & & & & \\
\hline$\Delta$ estimada $\left({ }^{\circ} \mathrm{C}\right)$ & 0 & $+1,75$ & $+2,15$ & $+2,65$ & -1 & & & & & \\
\hline Sin riesgo & 17.622 & 17.680 & 17.798 & 17.799 & 17.550 & 67,47 & 30 & 0,01 & $*$ & 0,88 \\
\hline Riesgo bajo & 79 & 56 & 0 & 0 & 65 & $-20,86$ & 6 & 0,09 & ns & 0,55 \\
\hline Riesgo moderado & 107 & 72 & 0 & 0 & 200 & $-50,65$ & 34 & 0,01 & $*$ & 0,89 \\
\hline Riesgo elevado & 10 & 11 & 21 & 20 & 5 & 4,04 & 11 & 0,04 & $*$ & 0,72 \\
\hline
\end{tabular}

Superficie expresada en unidades de $1.000 \mathrm{~km}^{2}$.

Se muestran los resultados de regresión lineal simple por ambiente de riesgo $(\alpha=0,05)$.

Cuadro 3. Análisis de sensibilidad del modelo de riesgo de establecimiento potencial de $L$. dispar a escala subcontinental. Sensitivity analyses of potential risk of establishment for $L$. dispar at subcontinental scale.

\begin{tabular}{|c|c|c|c|c|}
\hline \multirow{2}{*}{ Factor } & \multicolumn{2}{|c|}{ Índice S: riesgo elevado } & \multicolumn{2}{|c|}{ Índice S: riesgo moderado } \\
\hline & Actual & Escenarios futuros & Actual & Escenarios futuros \\
\hline Temperatura media enero & 28,67 & 20,41 & 7,62 & 2,59 \\
\hline Temperatura media julio & 0,81 & 19,67 & 8,95 & 9,59 \\
\hline Fase $2^{(*)}$ & 3,95 & 67,82 & 7,97 & 20,65 \\
\hline Índice de vegetación & 0,90 & 0,90 & 0,90 & 0,90 \\
\hline Fase $3^{(* *)}$ & 4,65 & 1,82 & 1,30 & 1,72 \\
\hline
\end{tabular}

Índice $\mathrm{S}=$ diferencia media en la superficie de riesgo entre el modelo modificado y el de referencia. Expresada en porcentaje (\%).

*Fase 2 = clasificación climática. **Fase 3 = Clasificación clima y hospedero susceptible.

Cuadro 4. Caracterización del riesgo de establecimiento potencial de L. dispar en las principales ecorregiones Argentinas, con cobertura vegetal susceptible y condiciones ambientales óptimas.

Risk characterization of establishment for $L$. dispar in the main ecoregions of Argentina with susceptible vegetation and optimal environmental conditions.

\begin{tabular}{|c|c|c|c|c|c|c|}
\hline \multirow{2}{*}{ Ecorregión } & \multicolumn{4}{|c|}{ Caracterización de ambientes de riesgo } & \multicolumn{2}{|c|}{$\operatorname{RMSE}^{(*)}$} \\
\hline & Sin riesgo & Bajo & Moderado & Elevado & $\square$ & $\mathrm{CV}$ \\
\hline Selva de yunga & 16.736 & 8.099 & 14.695 & 3.616 & 2,19 & 13 \\
\hline Chaco seco & 430.563 & 3.868 & 10.085 & 2.963 & 2,29 & 14 \\
\hline Delta e islas del Paraná & 24.784 & 22 & 12.996 & 70 & 1,80 & 16 \\
\hline Espinal & 286.980 & 3 & 4.515 & 631 & 2,11 & 15 \\
\hline Pampa & 334.865 & 4 & 26.051 & 19.977 & 1,79 & 7 \\
\hline Monte de llanuras y mesetas & 366.489 & 0 & 490 & 623 & 2,46 & 15 \\
\hline Bosques patagónicos & 64.647 & 488 & 330 & 22.137 & 2,75 & 23 \\
\hline
\end{tabular}

Superficie expresada en $\mathrm{km}^{2}$.

*RMSE = Raíz del error cuadrático medio de la temperatura media diaria entre el 1 de abril y el 31 de marzo. CV = Coeficiente de variación RMSE por ecorregión (expresado en \%).

región patagónica que para la pampeana, esto sería debido a la menor densidad de estaciones meteorológicas que presenta la región patagónica.

Los requerimientos de horas de frío (implícitos en el modelo fenológico) tendrían, a nivel regional, una mayor influencia sobre las fechas de eclosión de huevos, que so- bre la de eclosión de adultos. Esto puede deberse a que las mayores temperaturas imperantes durante el verano en los ambientes de menor latitud (figura 4), reducen el número de días calendario necesarios para completar el estado larval y pupal, disminuyendo en estos ambientes la variabilidad entre las fechas de eclosión de adultos (cuadro 5). 


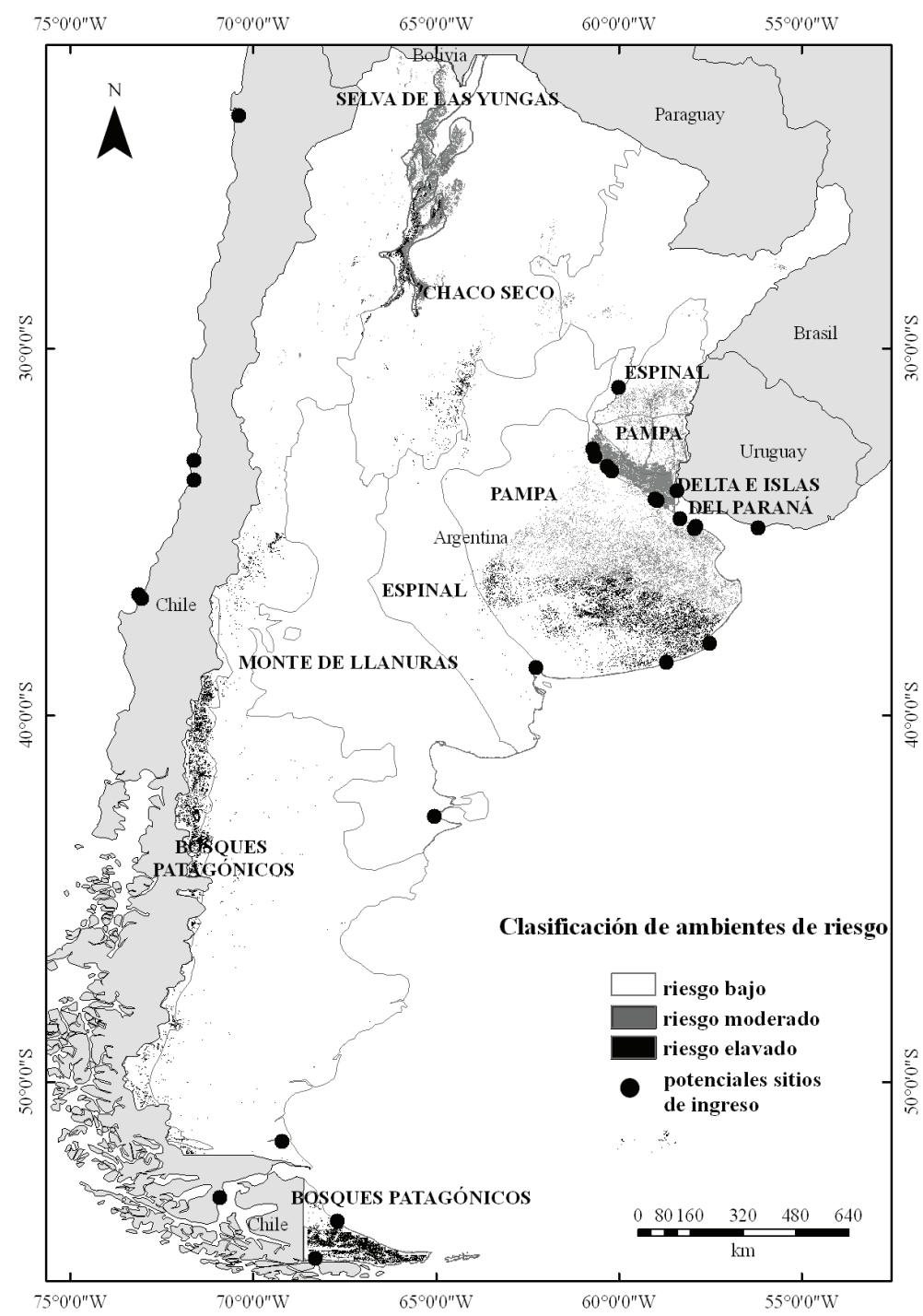

Figura 4. Caracterización de ambientes de riesgo para el establecimiento de $L$. dispar en Argentina según el modelo fenológico de Sheehan (1992).

Characterization of Argentina risk environments for establishment of L. dispar, according to the phenological model of Sheehan (1992).

Cuadro 5. Fecha estimada de eclosión del 50 \% de los adultos de L. dispar en Argentina según el modelo fenológico de Sheehan (1992). Estimated date of $50 \%$ adult emergence according to the phenological model of Sheehan (1992).

\begin{tabular}{|c|c|c|c|c|c|c|}
\hline \multirow{2}{*}{ Ecorregión } & \multicolumn{2}{|c|}{$\mathrm{N}^{\circ}$ días $\mathrm{T} .<5^{\circ} \mathrm{C}^{(*)}$} & \multicolumn{4}{|c|}{$\mathrm{N}^{\circ}$ días EC $50 \%$ adultos $^{(* *)}$} \\
\hline & $\bar{x}$ & $S$ & $\bar{x}$ & $S$ & IC $5 \%$ & IC 95\% \\
\hline Selva de yunga & 85,6 & 13,9 & 242 & 12,1 & 236 & 248 \\
\hline Chaco seco & 61,4 & 16,8 & 236 & 16,1 & 229 & 243 \\
\hline Delta e islas del Paraná & 50,0 & 5,4 & 231 & 19,3 & 222 & 240 \\
\hline Espinal & 70,0 & 19,4 & 228 & 15,6 & 221 & 235 \\
\hline Pampa & 37,6 & 14,6 & 225 & 18,5 & 216 & 234 \\
\hline Monte de llanuras y mesetas & 71,0 & 11,4 & 220 & 11,0 & 215 & 225 \\
\hline Bosques patagónicos Norte & 106,4 & 2,0 & 218 & 10,8 & 213 & 223 \\
\hline Bosques patagónicos Sur & 107,5 & 1,4 & 232 & 8,6 & 228 & 236 \\
\hline
\end{tabular}

* $\mathrm{N}^{\circ}$ días $\mathrm{T} .<5^{\circ} \mathrm{C}=\mathrm{N}^{\circ}$ días con temperatura media menor a $5^{\circ} \mathrm{C}$, entre el 1 de abril y el 30 de julio.

$* * \mathrm{~N}^{\circ}$ días EC $50 \%$ adultos $=\mathrm{N}^{\circ}$ días a eclosión del $50 \%$ de adultos a partir del 1 de julio. $\mathrm{n}=20$ por ecorregión $(\alpha=0,05)$. 


\section{DISCUSIÓN}

Estos resultados coinciden con los hallados mediante el software CLIMEX (Townsend et al. 2007), en la zonificación de ambientes de riesgo climático para el establecimiento de L. dispar a nivel subcontinental, y aportan información adicional al incorporar la distribución de coberturas boscosas/forestales susceptibles y el factor de riesgo de dispersión antrópica.

La reducción de la superficie bioclimáticamente óptima para el establecimiento de $L$. dispar en Sudamérica, a base de las estimaciones del IPCC para las próximas décadas, concuerda con los resultados hallados por Gevrey y Worner (2006), quienes determinaron que el factor que más contribuye a explicar los patrones de distribución actual de L. dispar, es la temperatura mínima anual. Sin embargo, no concuerdan con las estimaciones de Régnière et al. (2009), quienes estimaron que el cambio climático incrementaría el área boscosa susceptible de ser invadida por $L$. dispar, debido al probable aumento de la temperatura imperante durante el verano.

A nivel regional, las coberturas boscosas/forestales de la Patagonia presentan condiciones bioclimáticas óptimas para el establecimiento de $L$. dispar, pero se hallan alejadas de los puertos marítimos, mientras que los rodales de álamos y salicáceas del delta de Paraná, cercanos a los mismos, contarían con condiciones climáticas subóptimas para el desarrollo de esta plaga.

Vercken et al. (2011) sostienen que es necesaria la existencia de una densidad poblacional mínima, asociada a una superficie de bosque continuo, para asegurar la persistencia de la especie en el ambiente y su posterior dispersión a nuevas áreas. En tal sentido, algunas áreas bioclimáticamente favorables para el establecimiento de la especie, como por ejemplo la región pampeana, presentan un mosaico de pequeñas superficies forestales que sólo podrían sostener poblaciones dispersas de $L$. dispar, determinando así una baja probabilidad de ocurrencia de brotes poblacionales de la especie en el área.

El modelo fenológico utilizado en este trabajo fue diseñado para la raza europea de $L$. dispar, mientras que el cono sur presentaría un mayor riesgo de introducción de la raza asiática (COSAVE 2000). Considerando que los datos bioecológicos específicos de la raza asiática son escasos, se ha supuesto en este trabajo que existe una similitud suficiente entre ambas razas, que permita suponer que las áreas capaces de sostener poblaciones de la raza europea, lo serían también para la raza asiática. Sin embargo, algunas diferencias entre estas dos razas podría influir en la exactitud de riesgo estimado en este trabajo y por lo tanto, este análisis podría estar subestimando la magnitud del riesgo de establecimiento.

\section{CONCLUSIONES}

Aunque existieron diferencias significativas en la superficie clasificada como de mayor riesgo fitosanitario en las dos escalas de estudio (subcontinental vs. regional), se lograron identificar las principales áreas susceptibles de ser invadidas por $L$. dispar. Esto permitirá coordinar las tareas de monitoreo preventivo de la especie por los países del COSAVE.

Considerando los escenarios de calentamiento global propuestos por la IPCC, la cobertura boscosa susceptible a la colonización por la especie en Sudamérica, sería a futuro significativamente menor que en la actualidad.

\section{REFERENCIAS}

Andresen J, D McCullough, B Potter, C Koller, L Bauer, D Lusch, C Ramm. 2001. Effects of winter temperatures on gypsy moth egg masses in the Great Lakes region of the United States. Agricultural and Forest Meteorology 110: 85-100.

Bicheron P, M Leroy, C Brockmann, U Krämer, B Miras, C Vancutsem. 2006. GLOBCOVER: a $300 \mathrm{~m}$ global land cover product for 2005 using ENVISAT MERIS time series. 2nd International Symposium on Recent Advances in Quantitative Remote Sensing. Consultado 2 mar. 2012. Disponible en http://due.esrin.esa.int/prjs/Resul ts/131-176-131-25_2007510152728.pdf

Blanco P, W Sione, L Hardtke, H del Valle, P Aceñolaza, P Zamboni, G Heit, P Horak, P Cortese, R Moschini. 2010. Estimación espacial de variables climáticas en el territorio argentino mediante el uso de software libre. XIV Simposio Internacional de Selper. 8 - 12 Noviembre de 2010. Guanajuato México. s/p.

COSAVE (Comité de Sanidad Vegetal, UY). 2000. Estándar Regional en Protección Fitosanitaria. Sección III. Medidas Fitosanitarias. 3.10.2 Vigilancia de Lymantria dispar Linnaeus (Lepidoptera:Lymantriidae). Consultado 1 jun. 2011. Disponible en http://www.cosave.org.

Gadgil P, L Bulman, R Crabtree, R Watson, J O’Neil, K Glassey. 2000. Significance to New Zealand forestry of contaminants on the external surfaces of shipping containers. New Zealand Journal of Forestry Science 30: 341-358.

Gevrey M, S Worner. 2006. Prediction of Global Distribution of Insect Pest Species in Relation to Climate by Using an Ecological Informatics Method. Journal of Economic Entomology 99: 979-986.

Hijmans R, S Cameron, J Parra, P Jones, A Jarvis. 2005. Very high resolution interpolated climate surfaces for global land areas. International Journal of Climatology 25: 1965-1978.

Levine J, C D’Antonio. 2003. Forecasting biological invasions with increasing international trade. Conservation Biology 17: 322-326.

Liebhold A, J Elkinton, D Williams. 2000. What causes outbreaks of the gypsy moth in North America? Population Ecology: 257-266.

Liu C, M White, G Newell. 2009. Assessing the accuracy of species distribution models more thoroughly. XVIII World IMACS/MODSIM Congress. 13-17 julio 2009. Cairns, Australia. Consultado 2 may. 2014. Disponible en http:// mssanz.org.au/modsim09/J1/liu_c_J1a.pdf

Orán Cáceres J, M Gómez, W Plata. 2011. Posibilidades de aplicación de un análisis de sensibilidad secuencial a un modelo basado en técnicas SIG y evaluación multicriterio. Actas del XIII Conferencia Iberoamericana en Sistemas de Información Geográfica. 25-27 mayo de 2011. Toluca, México. 
Consultado 2 may. 2014. Disponible en http://www.inegi. org.mx/eventos/2011/conf_ibero/doc/ET4_18_ORAN.pdf

Ramirez J, A Jarvis. 2008. High resolution statistically downscaled future climate surfaces. International Center for Tropical Agriculture (CIAT); CGIAR Research Program on Climate Change, Agriculture and Food Security (CCAFS). Cali, Colombia.

Régnière J, N Vince, K Porter 2009. Climate suitability and management of the gypsy moth invasion into Canada. Biological Invasions 11:135-148

Sharov A, B Pijanowski, A Liebhold, S Gage. 1999. What affects the rate of gypsy moth (Lepidoptera: Lymantriidae) spread: winter temperature or forest susceptibility? Agricultural and Forest Entomology 1: 37-45.

Sheehan K. 1992. User's guide for GMPHEN: Gypsy Moth Phenology Model. Radnor, PA, USA. USDA Forest Service, General Technical Report 158. 29 p.

Soberón J, T Peterson. 2005. Interpretation of model of fundamental niches and species'distributional areas. Biodiversity
Informatics 2: 1-10

Tobin P, B Bai, D Eggen, D Leonard. 2012. The ecology, geopolitics, and economics of managing Lymantria dispar in the United States. International Journal of Pest Management 58:195-210.

Townsend P, W Richard, C Guojun. 2007. Modeled global invasive potential of Asian gypsy moths, Lymantria dispar. Entomologia Experimentalis et Applicata 125: 39-44.

Vanhanen H, Veteli T, Pailvinen S, Kellomaki S, P Niemala. 2007. Climate change and range shifts in two insect defoliators: gypsy moth and nun moth-a model study. Silva Fennica 41: 621-638.

Vercken E, A Kramer, P Tobin, J Drake. 2011. Critical patch size generated by Allee effect in gypsy moth, Lymantria dispar (L.). Ecology Letters 14: 179

Zlotina M, V Mastro, J Elkinton, D Leonard. 1999. Dispersal tendencies of neonate larvae of Lymantria mathura and the Asian form of Lymantria dispar (Lepidoptera: Lymantriidae). Environmental Entomology 28: 240-245. 
